the logical progression of hypotheses, the prediction of a possible new kind of observation, the design, construction and the use of an apparatus to search for the predicted phenomenon and the eventual success, the discovery. In recent times only the discovery of the $21-\mathrm{cm}$ hydrogen line fits this idealistic progression.

A more controversial point in Edge's paper concerns the reception of innovation by scientists. In some disciplines it seems that innovators are treated with apathy and hostility, forced to organise their own societies and journals, mainly because it was thought that the innovation tended to devalue the importance of the hard won skills and competence of established practitioners. Here astronomy seems to be anomalous, most innovators being happily supported and encouraged. Maybe this is because the astronomical innovators are not competing for scarce research resources against members of the established discipline. The pioneers of radioastronomy, for example, were supported by physics and engineering groups.

The final point concerns the strategy of a scientist when faced with a range of options for future research. To undertake an obvious experiment is to risk outright competition with other workers and possible loss of priority. Attempting a risky or speculative experiment, however, courts failure and also lessens enormously the audience which can appreciate its significance. Most scientists aim for the middle course, trying to prevent duplication and direct competition and trying to ensure success. Innovation, however, opens up a new era into which researchers rush, thus leading to a brief spell of outright competition and its concomitant secrecy.

Edge's paper helps lay to rest the scientists' impression of sociologists as a happy band rambling through science and occasionally stumbling on the obvious with shrieks of amazement. Does an understanding of sociology help the individual scientist? David Edge parries this question by answering that "if you want the (scientific) game to go better, then it is surely necessary to be clear about what kind of game it is". So we may conclude that the sociology of this subject is important, is useful, and should be more widely available to the scientific student.

\title{
Analysing tumour antigens
}

\author{
from Rod Langman
}

The Armand Hammer Cancer Workshop on Tumour Antigens was held at The Salk Institute, San Diego, on 26-29 September, 1977.

A SMALL group representing a wide range of interests met to discuss various aspects of tumour antigens. Classical serological techniques have provided the backbone of current knowledge, but as finer distinctions become necessary the limits of conventional technology in raising and absorbing antisera are being approached. This point was particularly well made during the discussion of chemically-induced tumour antigens. For example, A. Deleo (Sloan-Kettering) used a methyl cholanthrene-induced tumour of Balb/c mice (Meth-A), which had no detectable C-type virus antigen expression, to immunise syngeneic and semi-syngeneic mice. He found that what should be tumour-specific antisera had high levels of nonspecific activity as assayed on a wide range of normal and tumour tissues. These antisera were apparently loaded with antibody against.C-type

Rod Langman is Assistant Professor in the Laboratory of Developmental Biology at the Salk Institute. viral antigens which was produced when, under the stress of immunisation, endogenous C-type virus was induced. Despite extensive absorption the residual tumour-specific antibody still showed unexplained cross reactions that could not be further analysed. H. Festenstein (London Hospital) reported that the methyl cholanthreneinduced P815 tumour has apparent alterations in the $\mathrm{H}-2$ alloantigens, as detected by standard reference antisera. From this and a number of similar observations it was proposed that tumorigenesis may involve modulated expression of $\mathbf{H}-2$ alloantigens. While this interpretation was not widely accepted at the workshop, no competing explanation could be offered. Without better defined monospecific antisera it seems that antigens on chemicallyinduced tumours will remin elusive.

Although an obvious approach to preparing monospecific antibody, the Milstein-Galfré myeloma hybridisation technique has not yet been applied to tumour antigens. However, R. Levy (Stanford University) did introduce a variant of this idea when he described his adaptation of the Klinman spleenfragment method for preparing antibody to human leukaemic cells. First, large numbers of the patient's leu- kaemic cells were lightly fixed with glutaraldehyde; these were then used to immunise mice, and subsequently to assay antibody binding activity. When the spleens from immune mice were chopped into fragments and placed in microwell cultures, the antibody produced from each fragment could be assayed and from other studies it has been shown that under appropriate conditions each culture well contained one or fewer antibody-producing clones. The second key point of technique was the use of an iodinated rabbit anti-mouse immunoglobulin probe to detect antibody binding to the glutaraldehyde-fixed target cells. Under optimal conditions of spleen fragments culture and microassays for antibody binding, it was possible to obtain sufficient antibody from a single culture well to perform 2,000 assays. In practical terms it means that this technique can provide tumour-specific antibody (by selecting the appropriate culture well) which is tailored to suit the requirements of an individual patient, and in sufficient amounts to monitor accurately the numbers of leukaemic tumour cells during the course of therapy. In short, this technique could provide a major step forward in the management of tumours where chemotherapy is effective but must be closely regulated according to the tumour load.

Serological analysis of human melanoma antigens using autologous sera and cultured melanoma cells from tumour-bearing patients has revealed a variety of antigenic specificities (A. Deleo, Sloan-Kettening). A similar conclusion was reached by S. Ferrone (Scripps Clinic) who tested sera from melanoma patients against a panel of five cultured melanoma cell lines. In both studies there was no evidence of a common tumour-specific antigen as defined by patient sera. However, R. Reisfeld (Scripps Clinic) has shown that a single glycoprotein linked to

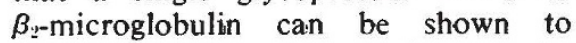
carry melanoma-specific antigens as defined by a heterologous rabbit antiserum. Putting the available data together, the tentative suggestion emerged that while one, or a few, molecules may carry several antigenic determinants which are tumour specific, there is substantial variation between individuals in the particular constellation of antigens present. In fact one wonders if the HLA system, in which three closely related molecular species carry a cluster of antigenic determinants which vary from one individual to the next, may be an important clue to introducing some order into this field. However, in the absence of genetics, improved chemistry is required to separate these related $\mathrm{mol}-$ ecules, and improved serology to elucidate the relationships. 